\title{
What can (mathematical) categories tell us about space-time?
}

\author{
Ko Sanders* \\ Institut für Theoretische Physik \\ Universität Leipzig \\ Brüderstraße 16 \\ D-04103 Leipzig
}

10 December 2015

\section{Introduction}

There are good reasons to believe that the classical structure of space-time, as it appears in general relativity, breaks down at small length scales of the order of the Planck scale [3]. This poses a problem in particular for any theory of quantum gravity, which should extend to such short length scales. Assuming that the classical concept of space-time (described as a manifold) is no longer viable as a fundamental concept in such a theory, one needs to explain how it emerges as an approximate concept in the appropriate (long distance) limit.

In order to understand what is required of such an explanation, it is necessary to have a good understanding of the classical structure of space-time. In this essay I will therefore focus on the concept of space-time as it appears in the axiomatic framework called locally covariant quantum field theory (LCQFT, [2]). This framework provides a language to formulate quantum field theories in the presence of gravitational background fields, and it encompasses some of the most precise physical theories that one can formulate without quantizing gravity. A key aspect of LCQFT is the way that it implements the conditions of locality and general covariance. These conditions have been crucial for a consistent, perturbative treatment of interacting quantum field theories [13], and they have even led to a perturbative description of quantum gravity [1].

The axiomatic framework of LCQFT is precise, flexible and quite general. On the one hand it is similar to algebraic quantum field theory (AQFT, [11]), an axiomatic framework for quantum field theories in Minkowski space, which has also been used by philosophers of physics because of its suitability for studying the foundations of quantum field theory [12].

*ko.sanders@physik.uni-leipzig.de 
On the other hand, it relies heavily on basic tools from category theory, which are used to formulate locality and general covariance. To the best of my knowledge, the use of category theory in this context has not been investigated by philosophers of physics before. Such an investigation is warranted, because it lies at the basis of some of the most profound results in LCQFT $[8,6]$, which address the question: "How do we know that a theory describes the same physics in all space-times?"

I will argue in this essay that the language of category theory, as used in LCQFT, carries more significance than a mere bookkeeping tool. It is used to give a precise and explicit statement of how a physical theory forms a model for modal logic, expressing a systems view of the world, and how space-time acts as an organizing principle that brings structure to our understanding of the world. This aspect of space-time is tightly related to locality and general covariance. However, it is not so directly related to the manifold structure, which means that it may not be lost in a quantum theory of gravity.

The argument that I present is to be viewed as a proposal, coming from a mathematical physicist. I will focus on philosophical aspects to the best of my ability. It is my intention that this essay serves as an invitation to the philosophical community to take up a more thorough investigation of the use of category theory in physical theories like LCQFT.

The structure of this essay is as follows. I will first review the basic concepts of LCQFT in Section 2. In Section 3, I will then review the argument that categories can be used in physics as models for modal logic, and how the categorical formulation of locality and general covariance in LCQFT can be viewed as an organizing principle. In Section 4, I will argue that this organizing principle has some non-trivial content. For that purpose I will review how it reflects on the identity problem ("How do we know that a theory describes the same physics in all space-times?"). In Section 5, I will formulate two proposals to characterize the notion of space-time in a general context, which should also apply to theories of (quantum) gravity. Section 6 contains some concluding remarks.

\section{Categories in locally covariant quantum field theory}

The framework of LCQFT, first introduced by Brunetti, Fredenhagen and Verch [2], is quite flexible and has since appeared in different variations, adapted to differing circumstances $[17,8,19,18,7]$. The subject of my argument is the use of category theory to implement locality and general covariance, which is common to all of these variations. In this section I will first present the main parts of the original framework, which are relevant for this essay, followed by a discussion of some of the possible variations. I will freely make use of basic notions from category theory (see [14] for a general reference).

In the spirit of AQFT [11], it is assumed that a quantum system can be described by a $C^{*}$-algebra $\mathcal{A}$ with unit, whose self-adjoint operators correspond to observable quantities. A state $\omega$ of the system is a normalized positive linear functional, and one may recover a Hilbert space formulation by using the GNS-construction. Instead of focusing on a single system, LCQFT uses many of them: one for each space-time. For this reason one introduces a category, $\mathrm{Alg}$, whose objects are $C^{*}$-algebras $\mathcal{A}$ with unit, and whose morphisms are 
injective $^{1 *}$-algebra homomorphisms $\alpha: \mathcal{A}_{1} \rightarrow \mathcal{A}_{2}$ which preserve the unit. The composition of morphisms is simply the composition of maps.

A LCQFT should describe a quantum field theory in every globally hyperbolic spacetime $M$. Here $M$ consists of a smooth, connected manifold $\mathcal{M}$ of dimension 4 , together with a smooth Lorentzian metric $g$, an orientation and a time-orientation, and $M$ admits a Cauchy surface. The collection of all globally hyperbolic space-times, where the theory can be localized, also forms a category, Loc, whose morphisms are isometric embeddings $\psi: M_{1} \rightarrow M_{2}$, which preserve the orientation, time-orientation, and the causal structure. The latter means in particular that any causal curve $\gamma$ in $M_{2}$ which connects points in $\psi\left(M_{1}\right)$ must lie entirely in $\psi\left(M_{1}\right)$.

Let us comment at this point on the relation between the category Loc and the notions of locality and general covariance in general relativity. It is often said that the symmetry group of general relativity is the diffeomorphism group of the manifold $\mathcal{M}$ underlying the space-time. In some sense, the category Loc refines this symmetry statement by including locality. Indeed, a general morphism $\psi: M_{1} \rightarrow M_{2}$ in Loc can be written as a composition $\psi=\iota \circ \phi$ of morphisms of a special kind. Here $\phi: M_{1} \rightarrow \psi\left(M_{1}\right)$ is a diffeomorphism, as used in the formulation of general covariance. $\iota: \psi\left(M_{1}\right) \rightarrow M_{2}$ corresponds to the canonical inclusion of a subset of the space-time $M_{2}$, expressing locality.

The fundamental definition of [2] states that a LCQFT is a covariant functor $\mathbf{A}$ : Loc $\rightarrow$ Alg. This means that it associates to every space-time $M$ in Loc a $C^{*}$-algebra $\mathbf{A}(M)$ in $\mathrm{Alg}$, and that it associates to every morphism $\psi: M_{1} \rightarrow M_{2}$ in Loc a morphism $\mathbf{A}(\psi): \mathbf{A}\left(M_{1}\right) \rightarrow \mathbf{A}\left(M_{2}\right)$ in $\mathbf{A l g}$. The algebra $\mathbf{A}(M)$ describes the quantum theory in the space-time $M$. The morphisms $\mathbf{A}(\psi)$ describe how an embedding of space-times gives rise to an embedding of the corresponding quantum systems. The requirement that $\mathbf{A}$ is a functor expresses the locality and general covariance of the theory. The simplest example of a LCQFT is the free scalar field with given mass and scalar curvature coupling [2].

On top of the requirement that a LCQFT is a functor $\mathbf{A}$, one may impose additional axioms. Let me mention here the time-slice axiom, which encodes the existence of a dynamical law, and local commutativity. A morphism $\psi: M_{1} \rightarrow M_{2}$ is called a Cauchy morphisms when its range $\psi\left(M_{1}\right)$ contains a Cauchy surface of $M_{2}$. Using a dynamical law it should then be possible to predict the behaviour of the system throughout $M_{2}$ using information available in $\psi\left(M_{1}\right)$. Accordingly, $\mathbf{A}$ is said to satisfy the time-slice axiom whenever $\mathbf{A}(\psi)$ is an isomorphism for all Cauchy morphisms $\psi$. For local commutativity one considers two morphisms $\psi_{1}: M_{1} \rightarrow M$ and $\psi_{2}: M_{2} \rightarrow M$ into the same target space-time $M$, such that ranges $\psi_{1}\left(M_{1}\right)$ and $\psi_{2}\left(M_{2}\right)$ are space-like separated. $\mathbf{A}$ is said to satisfy local commutativity whenever all operators in the range of $\mathbf{A}\left(\psi_{1}\right)$ commute with all operators in the range of $\mathbf{A}\left(\psi_{2}\right)$.

In addition to an algebra of observables, the specification of a quantum system requires the choice of a set of states. For a $C^{*}$-algebra one could take the set of all algebraic states, but in physical applications this set often contains many states with bad behaviour. This

\footnotetext{
${ }^{1}$ The injectivity assumption is not very well motivated, and it is known to fail for free electromagnetism, due to Gauss' law [18]. For this reason [18] suggested to drop it.
} 
suggests that the theory should also specify a subset of states, $\mathbf{S}(M)$ on the algebra $\mathbf{A}(M)$ for each space-time $M$. These sets of states are subjected to some conditions as well. E.g., they should be closed under taking mixtures (i.e. convex combinations) and for any morphism $\psi: M_{1} \rightarrow M_{2}$ the pull-back $\mathbf{A}(\psi)^{*} \omega$ of a state in $\mathbf{S}\left(M_{2}\right)$ should be a state on $\mathbf{S}\left(M_{1}\right)$. This means that the criterion that selects the sets of admissible states $\mathbf{S}(M)$ must be local and generally covariant as well. For free fields one typically uses the Hadamard condition.

Whereas Minkowski space-time has a group of symmetries, the structure of the category Loc is much more complicated. This has important consequences for the formulation of LCQFTs. Under quite general circumstances, there is no choice of a well-behaved natural state on all space-times [8], so there is no substitute for the Minkowski vacuum. Nevertheless, it is possible to treat interacting quantum field theories in curved space-times in a perturbative way (see [13] for a review). This requires the definition of renormalized (Wick) powers and time-ordered products of the quantum fields. The crucial insight here is that the freedom available in choosing a regularization and renormalization procedure is severely restricted by imposing the conditions of locality and general covariance. Proceeding in this way, mathematical physicists have also formulated perturbative Yang-Mills theories and even a perturbative description of quantum gravity in a categorical framework $[10,1]$. Note that this formulation of perturbative general relativity is by construction local and generally covariant.

As I mentioned above, the framework of LCQFT is quite flexible and has appeared in different variations. One type of variation is to replace the category Loc by another category BkGrnd, encoding the non-dynamical background structures for the theory. [17] uses a category of space-times with spin structure, in order to describe the free Dirac field. External source fields can also be included [19, 7], leading e.g. to a quite natural formulation of the Aharonov-Bohm effect in electromagnetism [18]. If one can include external source fields into the description of the background structure, one can also imagine removing the metric from it and including it with the dynamical fields in the category Phys. In that case one would replace the category Loc of globally hyperbolic space-times by a suitable category Man of manifolds in order to obtain a description of general relativity combined with other classical fields. I will address this approach, and any conceptual issues surrounding it, in Section 5.

In a similar way one can replace the target category without loosing any of the core ideas. [2] themselves used a category of more general topological *-algebras in the description of the free scalar field, which is convenient to make contact with the Wightman axioms. In a general analysis of locally covariant theories, Fewster and Verch [8] go much further and replace the target category by a quite general category Phys, which is restricted only by requiring a few basic properties. ${ }^{2}$ The objects of Phys are supposed to give a full description of the physical system. (They could consist e.g. of a $C^{*}$-algebra together with

\footnotetext{
${ }^{2}$ To be precise, the morphisms of Phys are required to be monomorphisms (which essentially means they are injective), and the category is required to have equalizers, intersections and unions in the categorical sense.
} 
a set of admissible states.) The consequences that [8] derives in this categorical approach rely on almost nothing except locality and general covariance, which lends them a very wide range of validity.

\section{Categories, modal logic and space-time}

From the review of the basic structure of LCQFT in Section 2 it is apparent that the language of category theory plays an important role. At the very least it is a convenient bookkeeping device, which exhibits the structure of locally covariant theories in a conceptually clear language. This is an advantage which has contributed to the recent theoretical development of perturbatively interacting quantum field theories in curved space-times. However, one may wonder whether the effectiveness of category theory has a deeper cause than mere bookkeeping. In this section I will argue that this is the case, by relating the categorical structure to a systems view of the world and the corresponding modal logic. I will then conclude that space-time in LCQFT acts as an organizing principle that brings structure to our understanding of the world.

Let us first follow [8] and consider the general target category, Phys, whose objects are a (mathematical) description of a physical system. Each system describes a part of the world. More precisely, we can think of the specification of an object in Phys as tantamount to the specification of (i) certain boundary conditions, like the space-time region and external fields, as well as (ii) the physical operations that can be performed on the system and (iii) the states that the system can be in. The morphisms in this category may be thought of as expressing a subsystem relation. The statement that Phys is a category imposes some relations on the morphisms, which can be seen as a consistency criterion that is needed to properly express the notion of subsystem. E.g., when $\alpha: P_{1} \rightarrow P_{2}$ and $\alpha^{\prime}: P_{2} \rightarrow P_{3}$ are morphisms, expressing how $P_{1}$ is a subsystem of $P_{2}$ and how $P_{2}$ is a subsystem of $P_{3}$, then $\alpha^{\prime} \circ \alpha$ expresses the way in which $P_{1}$ is a subsystem of $P_{3}$. Thus category theory provides a convenient way to express the systems view of the world.

It is a crucial part of the categorical language of LCQFT, and of theoretical physics in general, that theories can also describe situations which are not actually the case. The branch of logic which studies the truth values of statements and the validity of arguments that involve such situations is called modal logic [9]. Modal logic makes use of models in order to decide whether an argument is valid or not. I will now recall how the categorical structure of LCQFT makes it a model in the sense of modal logic [16].

The most common type of model makes use of possible worlds: complete alternatives for how things might be. Objects may or may not exist at a certain possible world and propositions and predicate statements may or may not hold. A typical system as described above can be embedded into a larger system, however, so it provides at best only an incomplete description of circumstances. For this reason it is appropriate to use a less wellknown model theory for modal logic, which makes use of incomplete sets of circumstances, so-called possibilities ([9] p.18-22). Due to its incompleteness, a possibility may not assign a truth value to every logical sentence. However, possibilities can be refined by extending the 
set of circumstances that it specifies, i.e. by extending the set of logical sentences which are assigned a truth value. This refinement is perfectly aligned with the structure of a category like Phys.

To be precise, we think of each object in Phys as a possible system that a given physical theory can describe, and which plays the role of a possibility in a model for modal logic. The morphisms of the category, which express the subsystem relation, then correspond to a refinement of such possibilities. I do not claim that every category must be a viable model for modal logic, but I do claim that this should hold for any category like Phys, which expresses the systems view of the world in a physical theory. The possibility semantics not only fits well with the systems view of the world, it also corresponds with experimental praxis: we would like to be able to make predictions for a certain laboratory experiment, without prescribing a complete set of circumstances for the entire world; making assumptions about the system in question should be enough.

Let me point out here that the extension of a system to a supersystem is usually not the only kind of refinement of possibilities that can occur in physics. Given a possible system, its state also specifies a set of circumstances (e.g. initial conditions). The states should therefore describe a model for modal logic in their own right. However, given the choice of a system, the state is supposed to describe a complete set of conditions, so the use of possible worlds is more appropriate in this case. Combing the two kinds of modalities, a possibility in general comprises the choice of a possible system together with the choice of a possible state. A refinement then consists of the embedding into a supersystem, together with an extension of the state.

I will address the distinction between the two modalities in Section 5, but let me note here that their interplay should be subject to certain restrictions, which are motivated by physics. It is clear that the refinements of a possibility are typically not unique. Concerning their existence we note that, if a system is known to be in a state which admits no extension to any supersystem, then one would expect the system to be the whole universe. Now let us consider the case where a state admits extensions to some of the possible supersystems but not to others. Implicitly, such a state contains information about the nature of any possible supersystems, as well as about the system itself. It is desirable to prohibit this kind of behaviour, because it indicates that we have made a poor choice in determining what information is needed to specify the system. In the context of LCQFT, the term "local physical equivalence" has been introduced to describe the assumption that essentially every state of a possible system can be extended to every supersystem [4].

Let me now come the use of functors in LCQFT. For a general morphism $\alpha: P_{1} \rightarrow P_{2}$, $P_{1}$ is a subsystem of $P_{2}$, so it describes less degrees of freedom. There may be many ways in which this can occur, e.g. when $P_{2}$ describes a system consisting of several fields and $P_{1}$ focuses only on one of these. The subsystems appearing in LCQFT are of a very specific type, however, because they are in the image of a functor $\mathbf{A}:$ Loc $\rightarrow$ Phys. We have seen in Section 2 that this functorial structure expresses the fact that the description of the system $\mathbf{A}(M)$ should depend in a local and covariant way on the space-time region $M$. The systems living in different space-times are therefore not independent. Because the principles of locality and general covariance are closely tied to the notion of space-time, 
the functorial structure is characteristic for how space-time enters into LCQFT.

In more general terms, if a physical theory is described by a category Phys of possible systems, the existence of the functor $\mathbf{A}$ (and the validity of other axioms of LCQFT) may be viewed as a condition on the category Phys. It states that a possible system $\mathbf{A}(M)$ can be determined from its localization region $M$, and the subsystem relation corresponds to a possible enlargement of the region $M$ (or rather, a morphism in Loc). LCQFT therefore stipulates in a precise and explicit way that space-time is to be viewed as an organizing principle, which brings additional structure to the collection of systems that a theory describes.

I will not attempt to place this statement in the appropriate philosophical or epistemological context - as a mathematical physicist this lies well outside my expertise. Instead I will address the possible criticism that the conclusions I have drawn about the notion of space-time in LCQFT are not very useful, because they rely only on elementary concepts of category and they do not contain any deep statements. In response it should be noted that the morphisms of the category Loc have a rich structure, which is much more complicated than the diffeomorphism group of a single manifold. I will illustrate in the next section how this structure can be used to analyse deep questions prove non-trivial results.

\section{On doing the same physics in all space-times}

In the general framework of LCQFT, Fewster and Verch [8] investigated the question: "What does it mean for a theory to describe the same physics in all space-times?" They point out that this is a difficult question, which is not adequately answered by specifying a Lagrangian. The question seems similar to a fundamental problem of identity which arises in philosophy: "How do we know that object A is the same as thing as object B, but at a different place and time and under different circumstances?" In everyday life we seem reasonably good at identifying objects, despite changing circumstances, but it is difficult to provide a clear theoretical explanation of why this is so. One of the arguments that may be presented in this context relates to the notion of space-time: two objects which occupy the same region in space-time are identical. Such arguments seem problematic in the context of classical field theory and quantum theory, let alone for theories in which space-time is not a fundamental concept. Nevertheless, it is interesting to note that the analysis of [8] also invokes space-time, because it makes essential use of the functorial structure of LCQFT, as I will now briefly review. (See [6] for a more detailed and accessible presentation.)

Let us consider two LCQFTs, A and B, given as functors from Loc to some category Phys. [8] call $\mathbf{A}$ a sub-theory of $\mathbf{B}$, whenever there is a natural transformation $\zeta: \mathbf{A} \rightarrow \mathbf{B}$ between the functors. This means that the following holds: for every space-time $M$ in Loc there is a morphism $\zeta_{M}: \mathbf{A}(M) \rightarrow \mathbf{B}(M)$ in Phys, and for every morphism $\psi: M_{1} \rightarrow M_{2}$ in Loc there holds:

$$
\zeta_{M_{2}} \circ \mathbf{A}(\psi)=\mathbf{B}(\psi) \circ \zeta_{M_{1}}
$$

The first condition states that on each space-time $M$, the system $\mathbf{A}(M)$ is a subsystem of $\mathbf{B}$, so it describes less degrees of freedom. The second condition essentially requires that 
the degrees of freedom which are left out are independent of the region of space-time that is being considered. Thus, it makes sense to restrict $\mathbf{B}\left(M_{2}\right)$ to $\mathbf{A}\left(M_{1}\right)$, without the need to prescribe the order in which the space-time and the degrees of freedom are being restricted.

Two theories are said to be equivalent when all the $\zeta_{M}$ are isomorphisms in Phys. In the language of category theory this means that $\zeta: \mathbf{A} \rightarrow \mathbf{B}$ is a natural isomorphism. When $\mathbf{B}$ is the same functor as $\mathbf{A}$, the natural isomorphisms form a group, which is interpreted as the global gauge transformations of the theory [5].

We emphasize that, from a physical point of view, it is not sufficient to require only the existence of the isomorphisms $\zeta_{M}$, without imposing Equation (1). [6] points out that this weaker condition is already satisfied in an analogous situation when $\mathbf{A}:$ BkGrnd $\rightarrow \mathrm{Alg}$ is a free scalar quantum field with an external source term $J$ (which is part of the background structure included in the objects of BkGrnd) and when B : BkGrnd $\rightarrow$ Alg is the same field with a source term $\lambda J$ for any constant $\lambda$. It is desirable that a non-trivial rescaling of the coupling to the external sources leads to an inequivalent theory, and this is achieved by also requiring (1). In fact, an analogous issue had already appeared in AQFT, where it has been recognized that the information of the theory is encoded in the subsystem relationship of the theory [11]. ${ }^{3}$ The main difference between AQFT and LCQFT is that the morphisms in the latter case have a much richer structure, because the category includes space-times with non-trivial gravitational background fields and non-trivial topology.

Fewster and Verch now propose the following line of reasoning. Suppose that we had a criterion to decide whether an LCQFT A describes the same physics in all space-times. Assume that two theories $\mathbf{A}$ and $\mathbf{B}$ satisfy this criterion, and that there exists a space-time $M$ such that $\mathbf{A}(M)$ and $\mathbf{B}(M)$ are equivalent. Then both theories should describe the same physics in all space-times, i.e. A should be equivalent to B. More technically, a criterion $\mathcal{C}$ on the class of LCQFTs has the Same Physics in All Space-times property (SPASs property) if and only if for any natural transformation $\zeta: \mathbf{A} \rightarrow \mathbf{B}$ between LCQFTs satisfying this criterion, $\zeta$ is a natural isomorphism as soon as $\zeta_{M}$ is an isomorphism for some $M{ }^{4}$

Perhaps surprisingly, [8] show that the axioms of LCQFT by themselves (including the time-slice axiom and local commutativity) are not sufficient to ensure that a theory describes the same physics in all space-times. For rather general LCQFTs A, [6] constructs a natural transformation $\zeta$ to another theory $\mathbf{B}$ such that $\zeta_{M}$ is an isomorphism for some space-times $M$, but not for all of them. This signals the need for an additional criterion $\mathcal{C}$ to supplement the axioms of LCQFT.

[8] goes on to propose a criterion $\mathcal{C}$ that does enjoy the SPASs property: dynamical locality. Once again this criterion makes essential use of the categorical structure of the theory, combined with the time-slice axiom and local commutativity. I will not give a full description of this criterion, but only indicate its main points, which should suffice to show

\footnotetext{
${ }^{3} \mathrm{In} \mathrm{AQFT}$, and also for $C^{*}$-algebraic LCQFTs, the local algebras are typically expected to be isomorphic to the unique, hyperfinite von Neumann factor of type $\mathrm{III}_{1}[12]$. The algebras by themselves, without their morphisms, therefore carry very little information.

${ }^{4}$ The requirement that the isomorphism $\zeta_{M}$ is part of a natural transformation $\zeta: \mathbf{A} \rightarrow \mathbf{B}$ prevents the isomorphism at $M$ from being an accidental pathology that does not respect locality and general covariance.
} 
that the categorical language of LCQFT allowed Fewster and Verch to prove non-trivial statements that address their identity problem.

Dynamical locality is defined for LCQFTs A whose target is the category $\mathrm{Alg}$ of $C^{*}$ algebras, and which satisfy local commutativity and the time-slice axiom. Using the timeslice axiom one can study how the system $\mathbf{A}(M)$ responds to a perturbation $\delta g$ of the metric, or of another external source field, supported in any compact region of space-time $M$. This response is encoded in the relative Cauchy evolution,

$$
\operatorname{rce}_{M_{2}, \delta g}: \mathbf{A}(M) \rightarrow \mathbf{A}(M),
$$

which is defined in [2]. Consider e.g. a morphism $\psi: M_{1} \rightarrow M$ and define the kinematic sub-algebra

$$
\mathbf{A}^{\operatorname{kin}}\left(\psi\left(M_{1}\right)\right):=\mathbf{A}(\psi)\left(\mathbf{A}\left(M_{1}\right)\right)
$$

of $\mathbf{A}(M)$. For any operator $X$ in $\mathbf{A}^{\text {kin }}\left(\psi\left(M_{1}\right)\right)$ and any perturbation $\delta g$ supported in a compact region that lies space-like to $\psi\left(M_{1}\right)$ one may show that

$$
\operatorname{rce}_{M_{2}, \delta g}(X)=0,
$$

due to local commutativity. This follows naturally from the localisation of $X$ and $\delta g$ and the fact that the theory has a dynamical law which respects the causal structure of space-time.

The idea of dynamical locality is that one can also determine the localization region of an operator $X \in \mathbf{A}(M)$ dynamically. The dynamical algebra $\mathbf{A}^{\text {dyn }}\left(\psi\left(M_{1}\right)\right)$ is essentially generated by operators $X \in \mathbf{A}(M)$ such that $\operatorname{rce}_{M_{2}, \delta g}(X)=0$ for all perturbations $\delta g$ with compact support space-like to $\psi\left(M_{1}\right)$. Dynamical locality requires that the kinematical and dynamical ways of localizing operators agree:

$$
\mathbf{A}^{\mathrm{dyn}}\left(\psi\left(M_{1}\right)\right)=\mathbf{A}^{\mathrm{kin}}\left(\psi\left(M_{1}\right)\right) .
$$

Dynamical locality has the SPASs property [8]. Thus, drawing a parallel to the philosophical identity problem, Fewster and Verch replace the claim that an object may be identified by the space-time region that it occupies, with a precise mathematical result that suggests that a quantum field theory may be identified when it is dynamically local, a property which depends on locality, general covariance, the causal behaviour of the theory and its response to perturbations of the metric (or other external source fields).

This is not the place to try and disentangle the role of the metric and the role of spacetime in this study, so let me conclude this section instead with the following remark. It is known that dynamical locality holds for the free scalar field, but it fails e.g. for free electromagnetism (once again due to Gauss' law) [6]. Indeed, Fewster and Verch do not claim that dynamical locality criterion is a fully satisfactory solution to their identity problem. However, their arguments do show that LCQFT provides a suitable language to address the problem and to prove non-trivial results that can contribute to its clarification. 


\section{On the notion of space-time}

I have argued that the use of category theory in LCQFT has a deeper meaning than mere bookkeeping, and that it allows to analyze deep questions. In this section I will discuss the question what the categorical point of view can tell us about the notion of space-time in the context of very general physical theories, including theories of quantum gravity.

We have seen in Section 3 that the systems view of the world is captured by the use of suitable categories Phys to describe physical theories. This immediately raises the question: Which categories are suitable for this purpose? In particular, one may wonder about the following issues: Should it be allowed that systems can be proper subsystems of themselves? Do we insist that the morphisms have to be monic (i.e. injective), or does that exclude Gauss' law $[8,18]$ ? Although these questions deserve further investigation, I will not dwell on them here. Let me turn instead to questions that concern the notion of space-time.

In LCQFT we assume the existence of a functor A : BkGrnd $\rightarrow$ Phys, where the category BkGrnd encodes the available background structure. This always consists of a globally hyperbolic space-time with a fixed metric, possibly with additional source fields. More general theories, such as pure general relativity (GR) or quantum gravity, will not have so much non-dynamical background structure. It seems natural to just assume some weaker structure and to replace the category Loc by, e.g., a category of manifolds, Man, or a category of discrete causal sets, as briefly suggested in [6]. I will now discuss a conceptual issue may arise in such approaches.

Suppose that there were a description of classical GR (in vacuum) as a functor GR : Man $\rightarrow$ Phys for a suitable category of (paracompact) manifolds and a category of physical systems. This means that the choice of a manifold $\mathcal{M}$ in Man determines a physical system. There is certainly no problem in the claim that $\mathcal{M}$ determines a set of solutions to Einstein's equation, or other mathematical, geometric objects. However, in order to do physics, the description of the system should normally include a class of observables, which may be more problematic. If we were to model the observables by test-functions (or test-tensors), as is usually done in field theory, then we would think of these observables as being localized in the region $\mathcal{M}$. However, the physical interpretation of this model is that we should set up an experimental device that is localized in $\mathcal{M}$. In order to do this, we need to have some way of physically identifying the points of $\mathcal{M}$. This is prohibited by the common understanding that the individual points of a space-time manifold in GR do not have any intrinsic physical meaning.

One viable attempt to circumvent this problem is to include non-dynamical background fields in the category Man, whilst keeping the metric dynamical. Note that not every manifold with a stress-tensor may admit a metric that solves Einstein's equations, due to the non-linearity of these equations. However, determining when the set of solutions is nonempty is a mathematical difficulty that will not concern us here. The downside to this approach is that it cannot capture the dynamics of all fields simultaneously.

The resolution can be sought in a change in the nature of the category Phys. Making use of partial observables, rather than observables, could circumvent the lack of local observables (see e.g. [15]). States then assign (expectation) values to suitable combinations 
of partial observables. This is not the place to develop this approach in detail, but it would be worthwhile to formulate it in a categorical setting, consistent with the systems view of the world. I expect that this should lead to a satisfactory formulation of classical GR and other classical Lagrangian field theories as a functor A : Man $\rightarrow$ Phys.

Because the objects of Man carry no structure beyond that of a manifold, I would argue that they encode the notion of space-time. Therefore, I would propose the following: We can characterize the notion of space-time mathematically by a functor $\mathbf{A}:$ Man $\rightarrow$ Phys. In this way, a space-time acts as an organizing principle, which brings structure to the theory that describes the systems in the category Phys. Theories which do not admit such a functor, do not have a classical space-time. This may apply to theories of quantum gravity. Of course they may still admit approximate notions of space-time, in some sense to be investigated.

To put this characterization of space-time into context, note that it suggests a shift in emphasis. The most fundamental aspect of space-time may not be that the objects of Man are manifolds, consisting of points with local charts etc. Instead, it might be how we can use the space-time: we use the manifolds to label systems in Phys, and we use the shrinking of the space-time region to label a corresponding subsystem relation.

Let me now try to rephrase the discussion above in the terms modal logic used in Section 3. There we have seen that the specification of a possibility consists of two parts: the specification of a possible system, which corresponded to a choice of space-time, together with the choice of a possible state on the system. In LCQFT, it is the interplay between these two types of modalities that seems to be characteristic for the way that space-time enters. Both the choice of system and the choice of state specify some circumstances (like boundary or initial conditions), but they are not on an equal footing. In LCQFT, the possible system must be chosen first, because it determines not only a set of boundary conditions for the system, but it determines the set of observables, which essentially make up the system. The choice of a state, on the other hand, does not alter the set of observables, but only assigns probability distributions to them. On top of that, the distinction between these two kinds of circumstances should be subject to certain conditions, as indicated in Section 3.

The formulation in terms of modal logic also applies to theories of the form $\mathbf{A}:$ Man $\rightarrow$ Phys. The category Phys may be quite different than in the case of LCQFT, e.g. it may only specify partial observables. Nevertheless, the choice of a possibility should still consist of two parts: the choice of a possible manifold and the choice of a state. This discussion leads to a second proposal on the characterization of space-time (perhaps in a somewhat generalized sense). It is closely related to the mathematical characterization above, but it is formulated in terms of the possibilities semantics: The notion of space-time (in a generalized sense) can be characterized as those circumstances that need to be specified in order to determine a system (e.g. the partial observables) of a theory. A state can be characterized as those circumstances that need to be specified in order to determine expectation values for combinations of partial observables. Theories for which the possibilities admit a precise distinction in these two aspects, admit a (generalized) notion of space-time. 


\section{Conclusions}

The original reason for [2] to introduce the language of category theory in LCQFT, was to express the requirements of locality and general covariance. However, I have argued that this language is appropriate quite generally to express a systems view of the world, because a physical theory corresponds to a category of possible systems, which can serve as a model of modal logic. The category of space-times Loc, and the requirement that theories are functors $\mathbf{A}:$ Loc $\rightarrow$ Phys, are a concrete expression of how space-time acts as an organizing principle that brings structure to our understanding of the world. I have also argued that the functorial structure of space-time may be highly non-trivial, because the source category may have many morphisms that lead to a rich structure. To illustrate this point I have used the work of Fewster and Verch [8], who use the structure of Loc to shed light on the deep question of identifying a quantum field theory in different space-times. However, I did not attempt to disentangle the roles of locality, general covariance, the metric and the causal behaviour of the theory in their argument.

In order to address the notion of space-time without a given metric, I have considered it in Section 5 as an organizing principle in the general context of the systems view of the world, as expressed in the language of category theory. In this context I have formulated two proposals to characterize the notion of space-time. The first is a mathematical condition: the existence of a functor A : Man $\rightarrow$ Phys. This raises the interesting question when such a functor exists, or, equivalently, under what conditions we can reconstruct the space-time from the category Phys. The second proposal is formulated in more abstract terms: spacetime can be characterized as the set of circumstances that specify a system of a theory. Although both formulations are somewhat analogous, I do not expect them to be fully equivalent, nor do I expect either of them to be the last word. I do hope, however, that they at least illustrate that the categorical language introduced by [2] is also suitable for the discussion of conceptual and philosophical questions, like those surrounding space-time and quantum gravity.

Along the way I have mentioned several additional questions. How can we split the possibilities of modal logic into possible systems and possible states? Which categories can act as a category of possible systems Phys? What does a category Phys look like that uses partial observables instead of observables? An investigation of these and of the proposals that I formulated will require input from philosophers of science as well as from mathematicians and physicists. It is the purpose of this essay to raise these questions to the philosophical community, and to promote a dialogue with the mathematics and physics communities.

\section{References}

[1] R. Brunetti, K. Fredenhagen and K. Rejzner, "Quantum gravity from the point of view of locally covariant quantum field theory", arXiv:1306.1058v4 [math-ph] (2013) 
[2] R. Brunetti, K. Fredenhagen and R. Verch, "The generally covariant locality principle - a new paradigm for local quantum field theory", Commun. Math. Phys. 237, 31-68 (2003)

[3] S. Doplicher, K. Fredenhagen, and J.E. Roberts, "Space-time quantization induced by classical gravity," Commun. Math. Phys. 172, 187-220 (1995)

[4] C.J. Fewster, "Quantum energy inequalities and local covariance II: Categorical formulation", Gen. Relativity and Gravitation 39 (2007), 1855-1890

[5] C.J. Fewster, "Endomorphisms and automorphisms of locally covariant quantum field theories", Rev. Math. Phys. 25, 1350008

[6] C.J. Fewster, "Locally covariant quantum field theory and the problem of formulating the same physics in all space-times", Philos. Trans. A 373 (2015), 20140238.

[7] C.J. Fewster and A. Schenkel, "Locally covariant quantum field theory with external sources", Ann. Henri Poincaré 16 (2015), 2303-2365

[8] C. Fewster and R. Verch, "Dynamical locality and covariance: What makes a physical theory the same in all spacetimes?", Ann. Henri Poincaré 13 (2012), 1613-1674

[9] G. Forbes, "The metaphysics of modality", Clarendon Press, Oxford (1985)

[10] K. Fredenhagen and K. Rejzner, "Batalin-Vilkovisky formalism in perturbative algebraic quantum field theory", Commun. Math. Phys. 317 (2013), 697-725

[11] R. Haag, "Local quantum physics", Springer, Berlin (1991)

[12] H. Halvorson, "Algebraic quantum field theory", with an appendix by M. Müger, In: J. Butterfield and J. Earman (Eds.), Philosophy of Physics (Handbook of the Philosophy of Science), Elsevier (2006)

[13] S. Hollands and R.M. Wald, "Quantum fields in curved spacetime", Phys. Rep. 574 (2015), 1-35

[14] S. Mac Lane, "Categories for the working mathematician", Springer, New York (1971)

[15] C. Rovelli, "Quantum Gravity", Cambridge University Press, Cambridge (2008)

[16] K. Sanders, "Aspects of locally covariant quantum field theory", PhD Thesis, University of York (2009), arXiv:0809.4828v1 [math-ph]

[17] K. Sanders, "The locally covariant Dirac field", Rev. Math. Phys. 22 (2010) 381-430

[18] K. Sanders, C. Dappiaggi and T.-P. Hack, "Electromagnetism, Local Covariance, the Aharonov-Bohm Effect and Gauss' Law", Commun. Math. Phys. 328, 625-667 (2014)

[19] J. Zahn, "The renormalized locally covariant Dirac field", Rev. Math. Phys. 26 (2014) 1330012 